\title{
Approximate Inertial Manifold-Based Model Reduction and Vibration Suppression for Rigid-Flexible Mechanical Arms
}

\author{
Lisha Xu, ${ }^{1,2}$ Hua Deng, ${ }^{1,2}$ Chong Lin, ${ }^{1,2}$ and Yi Zhang $\mathbb{D}^{1,2}$ \\ ${ }^{1}$ School of Mechanical and Electrical Engineering, Central South University, Changsha 410083, China \\ ${ }^{2}$ The State Key Laboratory of High Performance and Complex Manufacturing, Changsha 410083, China
}

Correspondence should be addressed to Yi Zhang; zhangyicsu@csu.edu.cn

Received 26 July 2021; Revised 25 August 2021; Accepted 12 September 2021; Published 31 October 2021

Academic Editor: Hang Su

Copyright $\odot 2021$ Lisha Xu et al. This is an open access article distributed under the Creative Commons Attribution License, which permits unrestricted use, distribution, and reproduction in any medium, provided the original work is properly cited.

\begin{abstract}
The dynamic characteristics of the mechanical arm with a rigid-flexible structure are very complex. The reason is that it is a complex DPS (distributed parameter system) with infinite dimension and nonlinearity in essence due to the rigid-flexible coupling. So, accurately positioning and controlling the rigid-flexible mechanical arms could be difficult. Therefore, a model reduction method of rigid-flexible mechanical arms based on the approximate inertial manifold is put forward. To repress the residual vibration of the end of the mechanical arm, a feedforward control strategy is designed. The high-dimensional solution of the vibration equation of the rigid-flexible mechanical arms is projected into the complete space composed of orthogonal decomposition modes. By using Galerkin's method, the system is simplified and the approximate solution is obtained through the interaction between high-order and low-order modes. The truncated finite mode is also used to construct a lowest-order dynamic model on the basis of approximate inertia manifold. Given the reduced-order rigid-flexible mechanical arms dynamic model, dynamic response analysis is conducted to optimize the target position error and end residual vibration. A limited number of sinusoidal signals approximately combine the input signal, by using the particle swarm optimization algorithm to optimize the input signal, and the amplitude of the sinusoidal signal is corrected. The simulation results depict the superiority of the proposed method, which greatly suppresses the end residual vibration of the mechanical arm and realizes the accurate positioning of the end of the mechanical arm. In addition, the hardware experimental device of the rigid-flexible mechanical arms is constructed, and the experimental verification of the above method is put into effect. The simulation results of angular displacement and end vibration of the reduced model are accordant which is shown by the experimental results of the hardware platform.
\end{abstract}

\section{Introduction}

A flexible mechanical arm has the advantages of a high weight ratio, low energy consumption, high efficiency, less inertia, and safe operation. At present, most researchers are focusing on flexible mechanical arms. However, less research involves rigid-flexible mechanical arms [1,2]. Rigid-flexible mechanical arms have stronger operability and versatility, higher payload, and more low total cost than traditional flexible mechanical arms $[3,4]$. They have been widely used in scientific fields, such as medicine and space [5-8]. Just like the cyber-physical systems, they also have more nonparametric uncertainties [9] and complex factors, such as dynamic/static friction, joint flexibility, structural resonance modes, and drive dynamics, than the traditional flexible mechanical arms. Given the complex coupling relationship between the state variables of the rigid-flexible mechanical arms [10], the difficulty of modeling and vibration suppression is greatly increased.

So far, many research studies on the modeling and deformation theory have been done by many researchers and achieved a series of results [11-13], for example, using finite element method, virtual modal method, and other methods to model flexible mechanical arm [14-17]. Various dimensionality reduction methods are used in this field. Researchers transform nonlinear partial differential equations from infinite dimension to finite dimension through models. Some of the commonly used methods include the 
traditional Galerkin's method (TGM) [18], inertial manifold method [19], intrinsic orthogonality decomposition method [20], and central manifold method [21]. When these methods are applied to nonlinear dynamic analysis, the question of how many modes should be included in the solution will arise. When insufficient modes are considered, the simplified system may not be topologically equivalent to the original system. By contrast, excessive considerations will bring difficulties to system analysis. In this way, the sensitivity of the solution to disturbance is the key factor affecting the accurate modeling of the nondynamic model.

This disturbance may come from the numerical error and mode truncation of the above method, and this slight disturbance may affect the system performance $[22,23]$. The approximate inertial manifold (AIM) has been widely used in recent years. The asymptotic properties of some infinitedimensional dissipative dynamic systems will evolve. They turn into a finite-dimensional compact set containing nonlinear phenomena called global attractors $[24,25]$. To the best of the researchers' knowledge, no research has introduced the AIM into flexible mechanical arms or rigidflexible coupling mechanical arms for dimensionality reduction.

Given that the flexible mechanical arm has underactuated variables, the controller for the underactuated variables of the system is difficult to design directly to converge in the study of position control. These difficulties lead to the residual vibration of the end of the mechanical arm in the movement process, which cuts down the accurate positioning of the end of the mechanical arm. Therefore, the residual vibration generated during the movement of the flexible mechanical arm needs to be suppressed, which is the key to achieving high-precision position control. At present, the main vibration suppression methods are active control and passive control $[26,27]$. Passive control is mainly achieved by adding a damping structure to the flexible structure. As early as the 1980s, the authors in [28] discovered that damping materials could be used to speed up the attenuation of residual vibration. They then proposed to increase the damping of the robot to achieve structural vibration suppression. However, this will change the structure of the mechanical arms. Active control includes feedforward control and closed-loop control [29-31]. Closed-loop control is the measurement of vibration signals by sensors and feedback to the control system. The control system outputs instructions to control the end vibration, including PID control, sliding mode control, robust control, state feedback control, fuzzy control, adaptive control, and neural network control [32-43]. The closed-loop control method mentioned above has a certain effect on the vibration restrain of the end of the flexible mechanical arm, but it needs to increase the sensor to measure the end deformation.

Moreover, constructing a complex feedback loop is necessary for the control, which makes its implementation in practical applications quite difficult. Feedforward control does not need to add additional sensors, and the control structure is very simple. Among them, input shaping is the most widely studied $[44,45]$. Yet, it needs to design the input shaping filter under some constraints. Many parameters are also needed to be considered. For example, one should consider the period, the number of pulses, and the amplitude of the pulse signal. To address the above problem, the authors in [46] proposed a feedforward control strategy that does not consider the input shaping filter to restrain the remains vibration of the long and flexible mechanical arm. They expressed the starting and braking part of the input signal as a Fourier series and optimized the coefficients of the Fourier series with the goal of zero remains vibration. Nevertheless, their methods only optimize the starting and braking parts of the input signal. The coefficients of the Fourier series were also modified through trial and error. Moreover, the selection of the higher mechanical harmonics is undetermined, and whether precise positioning can be achieved is not considered.

On the basis of the above problems, the infinite-dimensional solution of the vibration equation of the rigid-flexible mechanical arms is projected into the complete space formed by the POD (proper orthogonal decomposition) mode by using the AIM [47-49] in this study. According to AIM, the vibration equation of an infinite-dimensional rigid-flexible mechanical arm can be changed into a finite-dimensional equation to extract nonlinear phenomena. Then, the TGM is used to solve the simplified system to obtain the interaction between the highorder mode and the low-order mode. The proposed method reduces the free degree of the system and improves the calculation efficiency without significantly losing the accuracy of the solution comparing with the TGM. Given the reduced-order rigid-flexible mechanical arms dynamic model, dynamic response analysis is conducted to optimize the target position error and end residual vibration. The input signal is approximately combined by a limited number of sinusoidal signals. The NF (natural frequency) of the system determines the highest frequency of the sinusoidal signal, to optimize the input signal, and the amplitude of the sinusoidal signal is corrected using the particle swarm optimization (PSO) algorithm. The simulation results show that this method cannot only achieve precise positioning but also effectively suppress the residual vibration generated during the movement of the rigid-flexible mechanical arms.

The main dedications of this study are as follows:

(1) Based on the previous detailed description of the approximate inertial manifold theory, it is applied to the nonlinear model of rigid-flexible mechanical arms, and the model is simplified to obtain its lowest dimensional approximate model. This dimension reduction method not only retains the main characteristics of the original system but also reduces the degree of freedom of the system without significantly reducing the accuracy of the solution. Therefore, it brings great convenience for system analysis and subsequent controller design.

(2) To restrain the residual vibration of the rigid-flexible mechanical arms, a feedforward control strategy based upon PSO is proposed. The input shaping filter does not need to be considered. 
(3) The input signal is approximated by a combination of a finite number of sinusoidal signals, and the highest frequency of the sinusoidal signal is determined using the NF (natural frequency) of the system. The natural frequency of the system is obtained by physical analysis and simulation.

(4) According to the dynamic response of the rigidflexible mechanical arms, the PSO algorithm is also used to adjust the amplitude of the sinusoidal signal to achieve the purpose of optimizing the input signal. While realizing the precise positioning of the rigidflexible mechanical arms, it effectively suppresses the end residual vibration of the mechanical arm.

(5) For the lowest dimensional model of the rigidflexible mechanical arms, the feedforward control method is used, and the input signal based on particle swarm optimization is used as the input of the model controller. The results show that this method improves the calculation efficiency of the model, realizes the accurate positioning of the end of the flexible arm, and suppresses the residual vibration.

The other parts of this paper are arranged as follows. Part II builds the dynamics high-order model of rigid and flexible mechanical arm. Part III reduces the model based on approximate inertial manifold to obtain the lowest-order output model. Part IV conducts dynamic simulation analysis. An improved feedforward strategy about vibration suppression based upon PSO to optimize input signals is put forward in Part V. Part VI summarizes the achievements.

\subsection{Dynamic Modeling of the Rigid-Flexible Mechanical Arms.} The structure of the research object is shown in Figure 1. The rigid mechanical arm is connected to the fixed support base, and the flexible mechanical arm is connected to the end of the rigid mechanical arm by clicking the drive shaft. The following assumptions are made in modeling: the flexible mechanical arm can bend freely, after elastic deformation, the intersecting surface is vertical to the deformation axis, and the longitudinal deformation and gravitational potential energy are ignored in Figure 1, and the cyber-physical system composition diagram is depicted in Figure 2.

As depicted in Figure 1, the parameters are as follows:

$l_{1}$ is the length of the rigid mechanical arm

$l_{2}$ is the length of the flexible mechanical arm;

$O X Y$ is the inertial rectangular coordinate system with the base as the origin

$o_{1} x_{1} y_{1}$ is the local coordinate system of the rigid mechanical arm

$o_{2} x_{2} y_{2}$ is the local coordinate system of the flexible mechanical arm, and it turns with the turn of the mechanical arm

$\theta_{1}$ is the rotary angle of the coordinate system $o_{1} x_{1} y_{1}$ relative to the coordinate system $O X Y$

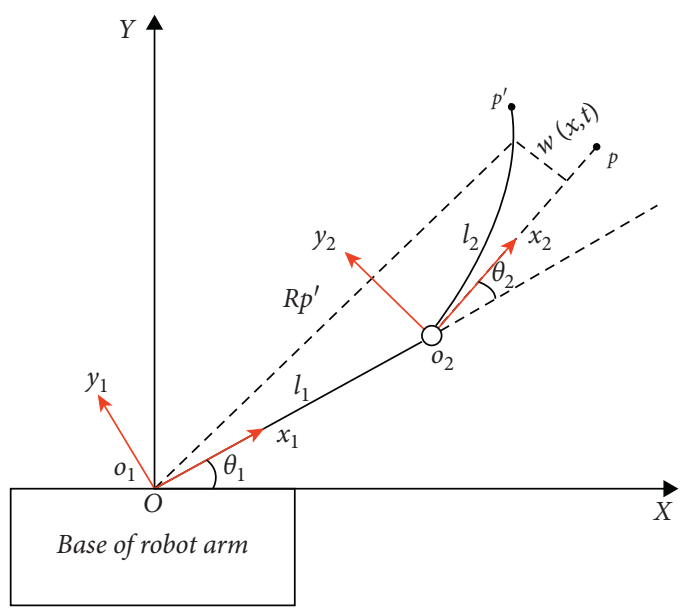

FIGURE 1: Structure diagram.

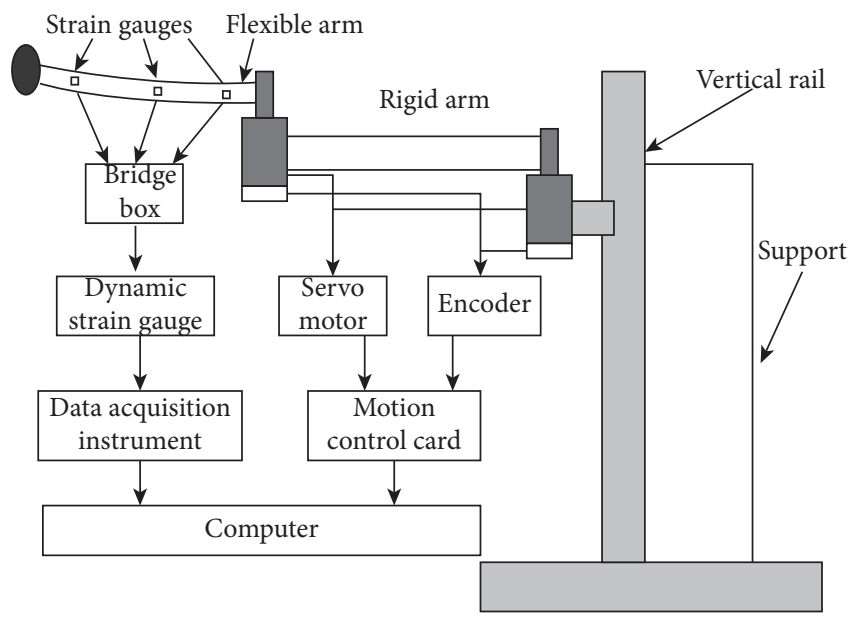

Figure 2: System composition diagram.

$\theta_{2}$ is the rotary angle of the coordinate system $o_{2} x_{2} y_{2}$ relative to the coordinate system $o_{1} x_{1} y_{1}$

According to the theory of Euler-Bernoulli beam and the results of reference [50], the vibration equation of the rigidflexible mechanical arm can be obtained as follows:

$$
\ell_{2} \ddot{w}(x, t)+2 \delta E I_{2} \dot{w}^{(4)}(x, t)+\ell_{2} E I_{2} w^{(4)}(x, t)=F(x, t),
$$

where $w(x, t)$ represents shift of the flexible mechanical arm at the deformed position.

The dynamic boundary constraints of the flexible mechanical arm are as follows:

$$
\begin{aligned}
F(x, t)= & \rho_{2}\left[l_{1} \sin \theta_{2} \dot{\theta}_{1}^{2}+l_{1} \cos \theta_{2} \ddot{\theta}_{1}^{2}-w(x, t)\left(\dot{\theta}_{1}+\dot{\theta}_{2}\right)^{2}\right] \\
& -M_{p}\left[l_{1} \cos \theta_{2} w\left(l_{2}, t\right) \ddot{\theta}_{1}-w\left(l_{2}, t\right)\left(\dot{\theta}_{1}+\dot{\theta}_{2}\right)^{2}\right. \\
& \left.+l_{2}\left(\dot{\theta}_{1}+\dot{\theta}_{2}\right)+l_{1} \dot{\theta}_{1}^{2} \sin \theta_{2}\right],
\end{aligned}
$$


where $F(x, t)=\ell_{2}\left[l_{1} \sin \theta_{2} \dot{\theta}_{1}^{2} \quad+l_{1} \cos \theta_{2} \ddot{\theta}_{1}^{2}-w(x, t)\left(\dot{\theta}_{1}+\right.\right.$ $\left.\left.\dot{\theta}_{2}\right)^{2}\right]-M_{p}\left[l_{1} \cos \theta_{2} w\left(l_{2}, t\right) \ddot{\theta}_{1}-w\left(l_{2}, t\right) \quad\left(\dot{\theta}_{1}+\dot{\theta}_{2}\right)^{2}+l_{2}\left(\dot{\theta}_{1}+\right.\right.$ $\left.\left.\dot{\theta}_{2}\right)+l_{1} \dot{\theta}_{1}^{2} \sin \theta_{2}\right]$.

The influence of joint friction factors should be considered to describe the dynamic model accurately. The four common static friction models are as follows: the Coulomb viscous friction model, Coulomb friction model, static friction Coulomb viscous friction model, and Stribeck friction model. The Coulomb viscous friction model adopts a continuous function to approximate the Stribeck friction model, which is conducive to the realization of the control of each joint motor. Therefore, the Coulomb viscous friction model is used to obtain the friction torque of each joint of the $\mathrm{n}$-degree-of-freedom mechanical arm as follows [51]:

$$
\nabla^{4}=\frac{\partial^{4}}{\partial x^{4}}
$$

where $i=1,2, f_{c i}$ represents the Coulomb friction torque coefficient, and $f_{v i}$ represents the viscous friction torque coefficient. In this way, the modified dynamic model of rigid-flexible mechanical arms can be obtained:

$$
\nabla^{4}=\frac{\partial^{4}}{\partial x^{4}}
$$

The coefficients of each matrix are given in Appendix A.

1.2. Model Reduction Based on Approximate Inertial Manifold. The approximate inertial manifold is essentially a Lipshitz manifold. When the time is large enough, the solutions of all the original equations will be attracted into a neighborhood of the manifold because its existence does not need the spectral interval condition. Using the approximate inertial manifold, it is possible for us to approximate and study the long-time dynamic behavior of the original equation with a finite-dimensional ordinary differential system; this ordinary differential equation is the approximate inertial form to be studied in this paper.

Equation (4) is a system of partial differential integral equations with nonlinear and strong coupling characteristics, and the exact analytical solution cannot be obtained directly. Therefore, dimensionality reduction is needed for the infinitedimensional nonlinear distributed parameter system.

The vibration equation (1) of the flexible mechanical arm can be rewritten as follows:

$$
\frac{\partial^{2} w(x, t)}{\partial t^{2}}=2 \delta \nabla^{4} \dot{w}(x, t)+\ell_{2} \nabla^{4} w(x, t)+f(x, t),
$$

where $\nabla^{4}=\partial^{4} / \partial x^{4}$ and $f(x, t)=F(x, t) / \ell_{2}$ is equivalent to the external force.

The linear operator of Equation (5) can be defined as $A w=\partial^{4} w / \partial x^{4}$. Following the TGM, in Equation (5), $w(x, t)$ is the displacement at the deformation point $x$ of the flexible mechanical arm, namely:

$$
w(x, t)=\sum_{i=1}^{\infty} \Gamma_{i}(x) q_{i}(t),
$$

where $\Gamma_{i}(x)$ is a characteristic function and $q_{i}=q_{i}(t)$ is an undetermined variable. Substituting (6) into (5) and considering the boundary conditions in (2), we have [50]

$$
\Gamma_{i}(x)=k_{i}\left(\sin \lambda_{i} x-\sinh \lambda_{i} x\right)-\cos \lambda_{i} x+\cosh \lambda_{i} x,
$$

where $\lambda_{i}$ is the eigenvalue, is the modal order, and

$$
k_{i}=\frac{\cosh \lambda_{i} l_{2}+\cos \lambda_{i} l_{2}}{\sinh \lambda_{i} l_{2}+\sin \lambda_{i} l_{2}} .
$$

The solution of equation (5) is expanded according to the orthogonal basis and using program of Galerkin, and the approximate solution of equation (5) can be obtained.

$$
\begin{aligned}
z= & \sum_{i=1}^{\infty} q_{i}(t) \cdot \Gamma_{i}(x)+E I_{2} \sum_{i=1}^{\infty} q_{i}(t) \frac{d^{4} \Gamma_{i}(x)}{d x^{4}} \\
& +\frac{2 \delta E I_{2}}{\ell_{2}} \sum_{i=1}^{\infty} \dot{q}_{i}(t) \frac{d^{4} \Gamma_{i}(x)}{d x^{4}}-f(x, t) .
\end{aligned}
$$

Let $U=\left[q_{1}, q_{2}, \ldots, q_{n}\right]^{T}$ represent the orthogonal projection in the Hilbert space $(\mathrm{H})$ onto the space strided over the first $n$ eigenfunctions of $A$ and $V=\left[q_{n+1}, q_{n+2}, \ldots, q_{\infty}\right]^{T}$ represent the rest, where $U$ is the low module subspace and $V$ is the high module subspace with infinite dimensions. It must be balance truncated, and the first $\mathbf{N}$ modes in all modes are used for the approximate solution of the model [22], which can be expressed by the inner product in $\mathbf{H}$, and then the following projections are obtained:

$$
\begin{aligned}
& \left(z, \Gamma_{k}\right)=0, \quad k=1,2, \ldots \ldots, n, \\
& \left(z, \Gamma_{k}\right)=0, \quad k=n+1, n+2, \cdots N .
\end{aligned}
$$

According to the above projections, we have

$$
\begin{gathered}
\ddot{U}+A_{1}(\dot{U}, \dot{V}, U, V)=0, \\
V+A_{2}(\dot{U}, \dot{V}, U, V)=0,
\end{gathered}
$$

where $A_{1}(\dot{U}, \dot{V}, U, V)$ and $A_{2}(\dot{U}, \dot{V}, U, V)$ are nonlinear functions of $\theta, q, \dot{\theta}, \dot{q}$.

By the spectral method combined with the Galerkin truncation criterion, the infinite-dimensional rigid-flexible mechanical arms system can be approximated to a thirddimensional model (i.e., $N=3$ ) after truncation [50]. Then, equation (6) can be written as follows:

$$
w(x, t)=\sum_{i=1}^{3} q_{i}(t) \Gamma_{i}(x) .
$$

Combining (4) and (12)-(14), a five-dimensional model of the rigid-flexible mechanical arms can be obtained as follows:

$$
M\left[\begin{array}{l}
\ddot{\theta} \\
\ddot{q}
\end{array}\right]+\left[\begin{array}{c}
F_{1}(\theta, q, \dot{\theta}, \dot{q}) \\
F_{2}(\theta, q, \dot{\theta}, \dot{q})
\end{array}\right]+\left[\begin{array}{c}
E_{1} \dot{\theta} \\
E_{2} \dot{q}+K q
\end{array}\right]=\left[\begin{array}{l}
u \\
0
\end{array}\right],
$$

where $M=M^{T}$ is the generalized symmetric inertia matrix, $\theta=\left(\theta_{1}, \theta_{2}\right)^{T}$ is the rotation angle vector of the mechanical 
arm joint, $q=\left(q_{1}, q_{2}, q_{3}\right)^{T}$ represents the modal value of flexible mechanical arm deformation, $u$ is the input resultant torque to the joint, $\left[F_{1}(\theta, q, \dot{\theta}, \dot{q}) \quad F_{2}(\theta, q, \dot{\theta}, \dot{q})\right]^{T}$ is a vector of nonlinear terms, $E_{1}=\operatorname{diag}\left(\mu_{1}, \mu_{2}\right)$ is the positive and definite damping matrix, and $E_{2}=\operatorname{diag}\left(\delta_{1}, \delta_{2}, \delta_{3}\right)$ is the structural damping matrix [52]. Equation (14) represents a five-dimensional strongly coupled nonlinear system based on generalized coordinates. The values of elements in the equation are shown in Appendix B.

Given that the nonlinear system (15) is a five-dimensional system with ten-order, it is a very high-order nonlinear system and difficult for system simulations, analyses, and controller design. Therefore, further truncation should be applied to (14). In equations (11) and (12), $U$ and $V$ contain low and high mode subspaces, and the modes in lower and higher subspaces can be thought of as "active" and "inactive," respectively [22]. Thus, a low-dimensional model may be obtained through the traditional Galerkin method by setting the first derivative of $\mathrm{V}$ and $\mathrm{V}$ in equation (12) to 0 then only the following equation can be obtained without considering equation (13)

$$
\ddot{U}+A_{1}(\dot{U}, U)=0 .
$$

Equation (15) means that the interaction between low modes and high modes is ignored when following the traditional Galerkin method. Nevertheless, on the basis of AIM theory, the higher-order modes of the flexible deformation displacement will soon decayed relatively to the lower-order modes under the action of viscous damping [22]. Thus, the derivatives of the higher-order mode $\mathrm{V}$ can be set to be zero, that is, $\ddot{V} \approx 0$ and $\dot{V} \approx 0$. From (13), a form of approximate inertial manifold can be built as

$$
A_{2}(\dot{U}, U, V)=0 .
$$

Substituting the solution of (17), i.e., $V \approx \Psi(\dot{U}, t U)$, into (16), (17) becomes

$$
A_{2}(\dot{U}, U, \Psi(\dot{U}, U))=0 .
$$

Equation (18) means that the low modes are used to capture the behavior of the high modes in equation (13), and the interaction between low modes and high modes is maintained. Substituting $V \approx \Psi(\dot{U}, U)$ and $\dot{V} \approx 0$ into (12), we have

$$
\ddot{U}+A_{1}(\dot{U}, U, \Psi(\dot{U}, U))=0 .
$$

Letting $n=1$ in equation (12) and substituting (18) and (19) into (13), a model with the lowest dimension (i.e., threedimension) can be obtained as follows:

$$
M\left[\begin{array}{l}
\ddot{\theta}_{1} \\
\ddot{\theta}_{2} 00 \\
\ddot{q}_{1}
\end{array}\right]+\left[\begin{array}{c}
J_{1}(\theta, q, \dot{\theta}, \dot{q}) \\
J_{2}(\theta, q, \dot{\theta}, \dot{q}) \\
J_{3}(\theta, q, \dot{\theta}, \dot{q}) \\
J_{4}(\theta, q, \dot{\theta}, \dot{q}) \\
J_{5}(\theta, q, \dot{\theta}, \dot{q})
\end{array}\right]+\left[\begin{array}{ccccc}
\mu_{1} & 0 & 0 & 0 & 0 \\
0 & \mu_{2} & 0 & 0 & 0 \\
0 & 0 & \delta_{1} & 0 & 0 \\
0 & 0 & 0 & \delta_{2} & 0 \\
0 & 0 & 0 & 0 & \delta_{3}
\end{array}\right]\left[\begin{array}{c}
\dot{\theta}_{1} \\
\dot{\theta}_{2} \\
\dot{q}_{1} \\
0 \\
0
\end{array}\right]+K\left[\begin{array}{c}
0 \\
0 \\
q_{1} \\
q_{2} \\
q_{3}
\end{array}\right]=\left[\begin{array}{c}
\tau_{1}-\tau_{f 1} \\
\tau_{1}-\tau_{f 2} \\
0 \\
0 \\
0
\end{array}\right],
$$

where

$$
\begin{aligned}
J_{1}(\theta, q, \dot{\theta}, \dot{q})= & -\left[\frac{1}{2} \ell_{2} l_{1} l_{2}^{2} \sin \theta_{2}+\ell_{2} l_{1} \cos \theta_{2} \sum_{j=1}^{3} h_{j}(1) q_{j}(t)\right] \dot{\theta}_{2}^{2}-\left[\ell_{2} l_{1} l_{2}^{2} \sin \theta_{2}+\ell_{2} l_{1} \cos \theta_{2} \sum_{j=1}^{3} h_{j}(1) q_{j}(t)\right] \dot{\theta}_{1} \dot{\theta}_{2} \\
& -M_{p}\left(l_{1} \cos \theta_{2} \sum_{i=1}^{3} \int_{0}^{l_{2}} \Gamma_{i}(x) q_{i}(t) d x+2 l_{1} l_{2} \sin \theta_{2}\right) \\
& \dot{\theta}_{2}^{2}-\left[2 \ell_{2} L_{1} \sin \theta_{2} \sum_{j=1}^{3} h_{j}(1) \dot{q}_{j}(t)-2 \ell_{2} L_{2} \sum_{j=1}^{3} q_{j}(t) \dot{q}_{j}(t)\right]\left(\dot{\theta}_{1}+\dot{\theta}_{2}\right) \\
& +M_{p}\left(2 l_{1} \cos \theta_{2} \sum_{i=1}^{3} \int_{0}^{l_{2}} \Gamma_{i}(x) q_{i}(t) d x+2 l_{1} l_{2} \sin \theta_{2}\right) \dot{\theta}_{1} \dot{\theta}_{2}, \\
J_{2}(\theta, q, \dot{\theta}, \dot{q})= & {\left[\frac{1}{2} \ell_{2} l_{1} l_{2}^{2} \sin \theta_{2}+\ell_{2} l_{1} \cos \theta_{2} \sum_{j=1}^{3} h_{j}(1) q_{j}(t)\right] \dot{\theta}_{1}^{2}+2 \ell_{2} l_{2}\left(\dot{\theta}_{1}+\dot{\theta}_{2}\right) }
\end{aligned}
$$




$$
\begin{aligned}
& \left(\sum_{j=1}^{3} q_{j}(t) \dot{q}_{j}(t)+M_{p}\left(l_{2}^{2}+\sum_{j=1}^{3} h_{j}(1) q_{j}(t) \cdot \sum_{j=1}^{3} q_{j}(t) \dot{q}_{j}(t)\right)\right) \\
& +M_{p}\left(l_{1} \cos \theta_{2} \sum_{j=1}^{3} h_{j}(1) q_{j}(t)+l_{1} l_{2} \sin \theta_{2}\right) \dot{\theta}_{1}^{2}, \\
J_{i+2}(\theta, q, \dot{\theta}, \dot{q})= & -\ell_{2}\left(\dot{\theta}_{1}+\dot{\theta}_{2}\right)^{2} q_{i}+\ell_{2} l_{2} \dot{\theta}_{1}^{2} \sin \theta_{2} \cdot h_{i}(1), \quad i=1,2,3 .
\end{aligned}
$$

And solution (18) gives the following:

$$
\begin{aligned}
q_{2}= & \tau_{2} m_{33}-\left\{\left[2 m_{33} \ell_{2} l_{2}\left(\dot{\theta}_{1}+\dot{\theta}_{2}\right) q_{1}-k_{3} m_{23}\right] \dot{q}_{1}+\ell_{2} l_{1} \cos \theta_{2} \cdot h_{1} \dot{\theta}_{1}^{2} m_{33}-\left(\delta_{3}-\ell_{2}\left(\dot{\theta}_{1}+\dot{\theta}_{2}\right)^{2} m_{23}\right) q_{1}\right\} \\
& +\ell_{2} l_{1} \cos \theta_{2} \cdot h_{3} \dot{\theta}_{1}^{2} m_{33} \cdot \frac{\ell_{2} l_{2} \dot{\theta}_{1}^{2} \sin \theta_{2} \cdot h_{3}}{\ell_{2}\left(\dot{\theta}_{1}+\dot{\theta}_{2}\right)^{2}-\delta_{5}} \\
& +\left[k_{2} \dot{\theta}_{2}+\delta_{2} \theta_{2}+\frac{1}{2} \ell_{2} l_{1} l_{2}^{2} \sin \theta_{2} \dot{\theta}_{1}^{2}\right] m_{33}-\frac{\ell_{2} l_{2} \dot{\theta}_{1}^{2} \sin \theta_{2} \cdot h_{1} m_{23}}{\ell_{2} l_{1} \cos \theta_{2} \cdot h_{2} \dot{\theta}_{1}^{2} m_{33}}, \\
q_{3}= & \tau_{2} m_{33}-\left\{\left[2 m_{33} \ell_{2} l_{2}\left(\dot{\theta}_{1}+\dot{\theta}_{2}\right) q_{1}-k_{3} m_{23}\right] \dot{q}_{1}+\ell_{2} l_{1} \cos \theta_{2} \cdot h_{1} \dot{\theta}_{1}^{2} m_{33}-\left(\delta_{3}-\ell_{2}\left(\dot{\theta}_{1}+\dot{\theta}_{2}\right)^{2} m_{23}\right) q_{1}\right\} \\
& +\ell_{2} l_{1} \cos \theta_{2} \cdot h_{2} \dot{\theta}_{1}^{2} m_{33} \cdot \frac{\ell_{2} l_{2} h_{2} \dot{\theta}_{1}^{2} \sin \theta_{2}}{\ell_{2}\left(\dot{\theta}_{1}+\dot{\theta}_{2}\right)^{2}-\delta_{4}} \\
& +\left[k_{2} \dot{\theta}_{2}+\delta_{2} \theta_{2}+\frac{1}{2} \rho_{2} l_{1} l_{2}^{2} \dot{\theta}_{1}^{2} \sin \theta_{2}\right] m_{33}-\frac{\rho_{2} l_{2} \dot{\theta}_{1}^{2} \sin \theta_{2} \cdot h_{1} m_{23}}{\rho_{2} l_{1} \cos \theta_{2} \cdot h_{3} \dot{\theta}_{1}^{2} m_{33}},
\end{aligned}
$$

where $m_{23}=\ell_{2} \int_{0}^{l_{2}} x \cdot \Gamma_{1}(x) \mathrm{d} x+M_{p} l_{2} \int_{0}^{l_{2}} \Gamma_{3}(x) \mathrm{d} x \quad$ and $m_{33}=\ell_{2} \int_{0}^{l_{2}} \Gamma_{1}(x) \mathrm{d} x$.

Finally, substitute $w(x, t)=\Phi_{1}(x) q_{1}+\Phi_{2}(x) q_{2}+\Phi_{3}$ $(x) q_{3}$ and $w(x, t)=\Phi_{1}(x) q_{1}+\Phi_{2}(x) q_{2}+\Phi_{3}(x) q_{3}$ into equation (14) to get the low-dimensional output model of rigid-flexible mechanical arms as follows:

$$
w(x, t)=\Gamma_{1}(x) q_{1}+\Gamma_{2}(x) q_{2}+\Gamma_{3}(x) q_{3} .
$$

\section{Dynamic Simulations}

2.1. Model Simulation and Analysis. The initial state of the rigid and flexible mechanical arms is assumed in a horizontal position; the basic initial parameters are as follows: $\theta_{1}=\theta_{2}=q_{1}=q_{2}=q_{3}=0$. The joint input torque is $\tau_{1}=\tau_{2}=0.412 \mathrm{~N} / \mathrm{m}$. The length, width, and height of the flexible mechanical arm are $300 \mathrm{~mm}, 20 \mathrm{~mm}$, and $15 \mathrm{~mm}$, respectively. Other parameters are shown in Table 1 . The relationship between modal and energy is obtained by simulation as depicted in Figure 3. The first-order mode accounts for $94.12 \%$ of the total energy, and the first threeorder modes account for $99.78 \%$ of the total energy. This finding shows that the accuracy of the first three models is very high, but only taking the first mode is not enough to ensure the accuracy of the model.
2.2. Free-Response Dynamic Simulations. When the input torque is 0 and only the initial state is given, the response results of the approximate model obtained by the spectral method and the exact solution are compared. The initial time is $t_{0}=0$, and the end time is $t_{f}=10 \mathrm{~s}$. The initial state of the system is $\theta_{1}=0, \theta_{2}=\pi / 4, \dot{\theta}_{1}=\dot{\theta}_{2}=0, q_{i}=0$, and $i=1,2,3$. The initial value of the first derivative of all other generalized variables is 0 . The fourth-order Runge-Kutta formula is used to solve the AIM-based low-order model and the TGMbased truncated third-order model. The simulation results are depicted in Figures 4-7.

In the free-response without input torque, $\theta_{1}(t)$ and $\theta_{2}$ $(t)$ are finally stabilized, and the mutual influence between the rigid-flexible coupling can be obtained. The end deformation of the lowest-order model based on AIM is almost similar to that of the truncated flexible model based on TGM, as shown in Figures 4 to 6 . They are all stabilized at zero in the end because of the features of structural damper of the mechanical arm itself. The simulation results of the first-order system and the third-order system after using the AIM approximation are the same, as shown in Figure 7. This conclusion can further prove that the AIM method is effective for model dimensionality reduction and can retain the main characteristics of the original system dynamics. Compared with the TGM-based truncation method, the lowest-order system based on AIM has a 
TABLE 1: Other parameter values of the rigid-flexible mechanical arms.

\begin{tabular}{lcc}
\hline Parameter name & Rigid mechanical arm & Flexible mechanical arm \\
\hline Length $(\mathrm{m})$ & $L_{1}=0.33$ & $L_{2}=0.30$ \\
Moment of inertia $\left(\mathrm{kg} \cdot \mathrm{m}^{2}\right)$ & $J_{1}=0.0812$ & $J_{2}=0.138$ \\
Line density $\left(\mathrm{kg} \cdot \mathrm{m}^{3}\right)$ & & $\ell_{2}=0.4865$ \\
Elastic modulus $\left(\mathrm{N} \cdot \mathrm{m}^{2}\right)$ & & $E I_{2}=26.055$ \\
End mass $(\mathrm{kg})$ & $M_{t}=0.721$ & $M_{p}=0.5$ \\
\hline
\end{tabular}

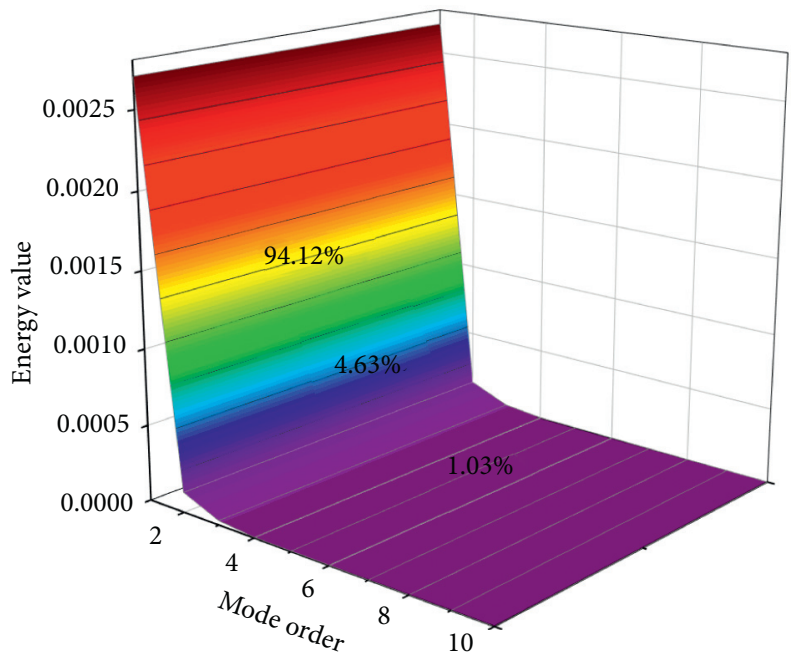

FIgURE 3: Relationship of modes and energy.

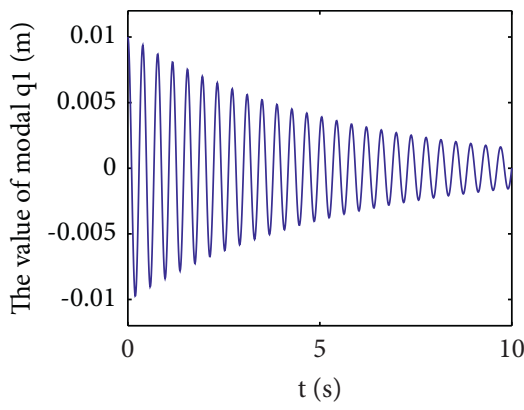

(a)

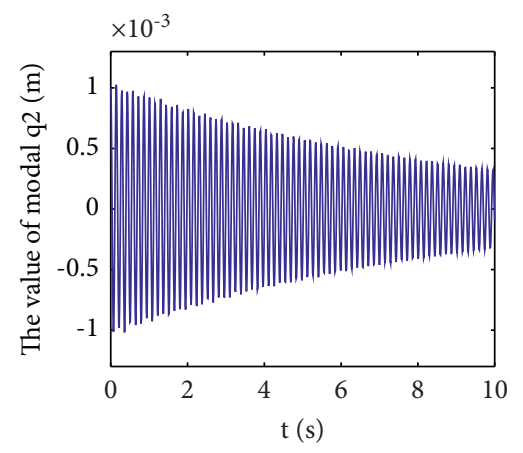

(b)

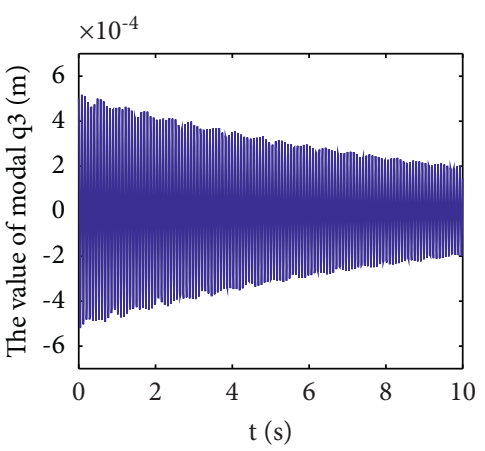

(c)

FIgURe 4: Generalized coordinate curve of flexible deformation: (a) $q 1$; (b) $q 2$; (c) $q 3$.

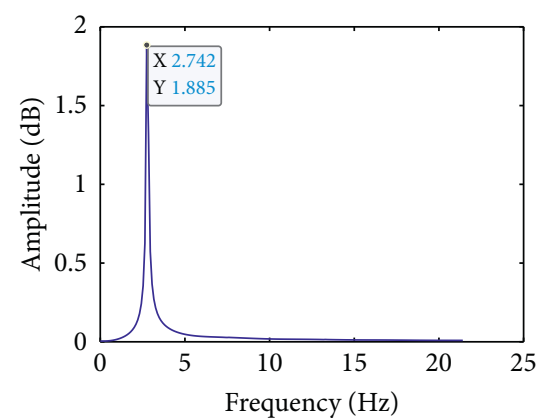

(a)

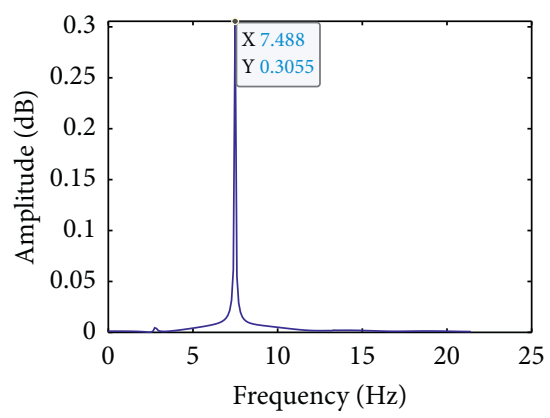

(b)

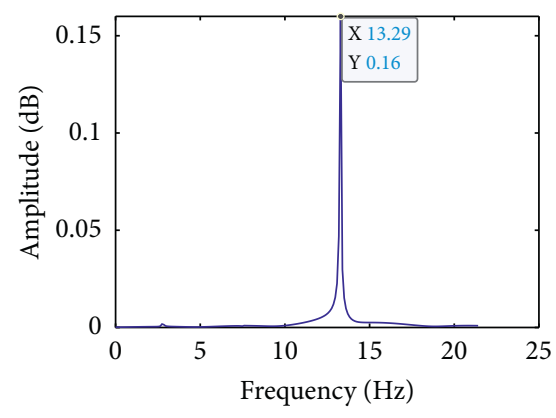

(c)

FIGURE 5: Spectrum of flexible generalized coordinates: (a) spectrum of $q_{1}$; (b) spectrum of $q_{2}$; (c) spectrum of $q_{3}$. 


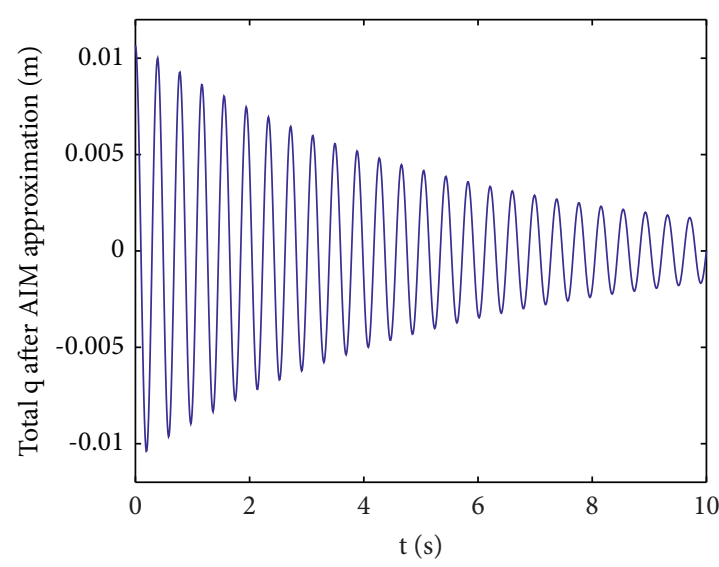

(a)

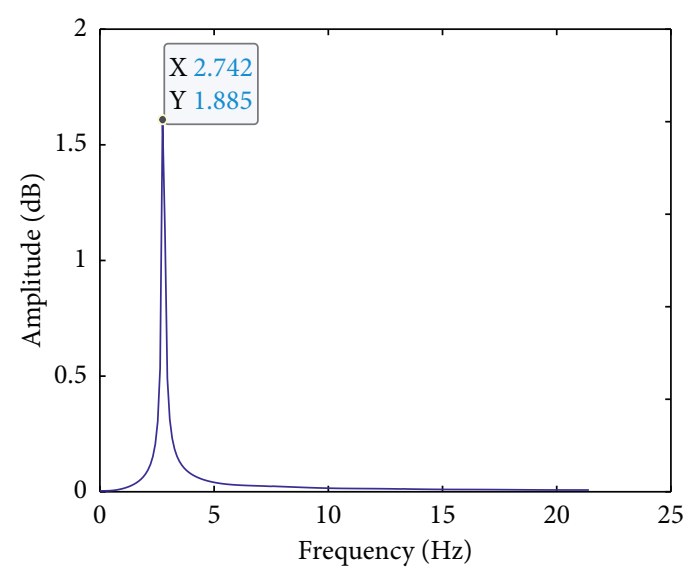

(b)

Figure 6: (a) Curve (q) of AIM; (b) spectrum (q) of AIM

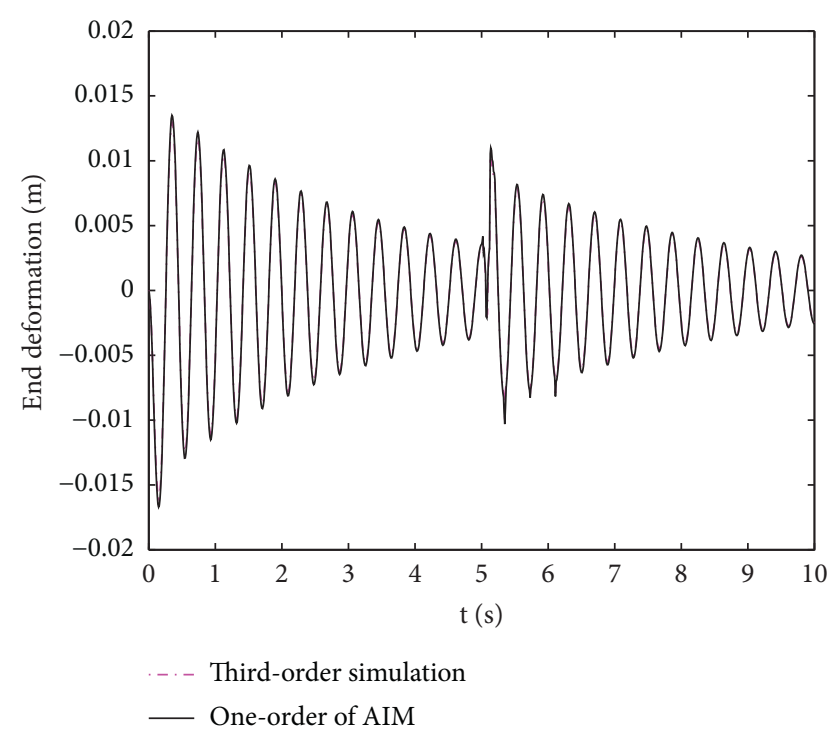

FIGURE 7: End vibrations with the first-order model and the thirdorder model.

smaller amount of calculation; according to this method, it is very convenient to analyze the system and design the controller.

\section{Vibration Suppression Based on PSO}

The AIM approximation model is used for numerical calculation in this study, and the function coefficients of the approximate input signal are optimized to suppress the end vibration. Firstly, a series of continuous sinusoidal functions approximate the input signal. The selection of the numbers of the approximation function is determined by the natural frequency, which is applied to the flexible mechanical arm to minimize the target position error and the displacement of end vibration. Then, using the PSO algorithm, the coefficients of each input function are optimized without considering the input shaping filter $[44,45]$. In this way, the calculation efficiency of complex dynamic response is significantly improved, and the residual vibration is minimized when the target position is reached. The control structure diagram is depicted in Figure 8.

3.1. Optimization-Based Vibration Suppression and Precise Positioning. In Figure 1, the input signals acting on Joint 1 and Joint 2 are represented by trapezoidal signals, as shown in Figure 9. The expression is shown as follows.

$$
u(t)= \begin{cases}\frac{A t}{\varpi}, & 0 \leq t<\omega, \\ A, & \varpi \leq t<(T-\Phi), \\ \frac{A(T-t)}{\varpi}, & (T-\varpi) \leq t<T .\end{cases}
$$

It is assumed that the operating cycle of rigid-flexible mechanical arms is $T$. Therefore, the signal $u(t)$ can be approximately expressed by a linear combination of infinitely many sinusoidal functions of different frequencies on $[0, T]$ and as depicted by the following formula:

$$
u(t)=\frac{k_{0}}{2}+\sum_{n=1}^{\infty} k_{n} \sin w_{n} t,
$$

where $k_{n}$ is the coefficient to be optimized and $w_{n}=2 \pi n / T$ is the angular frequency. Theoretically, the greater the value of $n$, the higher the fitting accuracy of the function. The time acting on the signals of Joint 1 and Joint 2 is taken as $4 \mathrm{~s}$, and $d$ is taken as $0.1 \mathrm{~s}$. The fundamental frequency is $2 \pi /$ $T$. When $n$ is taken as $11, w_{n}$ is close to the natural frequency of the mechanical arms (as shown in Figure 6(b)). In this case, the end vibration will not be suppressed but increased. Thus, $n$ is selected as 9 . The parameters of the input signal of Joint 1 to be optimized are $k_{10}, k_{11}, k_{12}$, $\ldots \ldots, k_{19}$. The parameters of the input signal of Joint 2 to be optimized are $k_{20}, k_{21}, k_{22}, \ldots \ldots, k_{29}$. These parameters are combined as follows: 


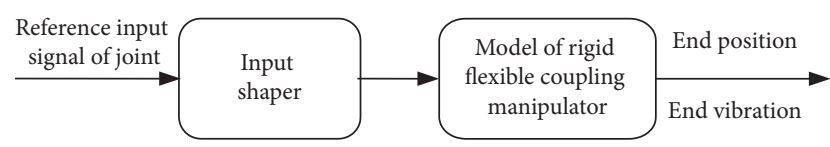

Figure 8: The control structure diagram of feedforward.

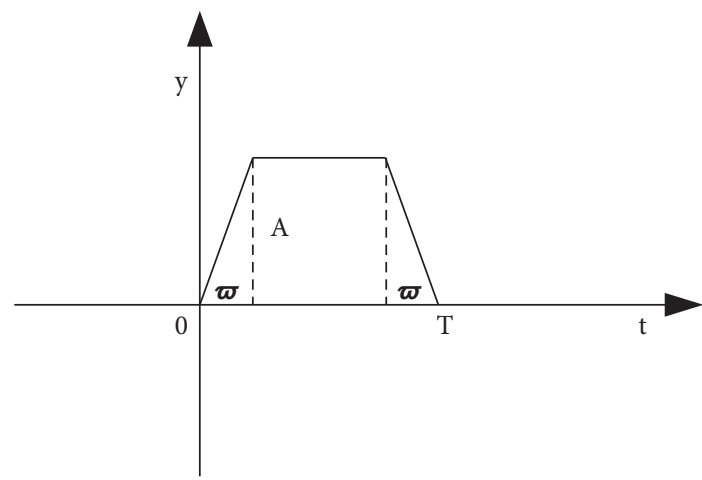

Figure 9: Trapezoidal signal.

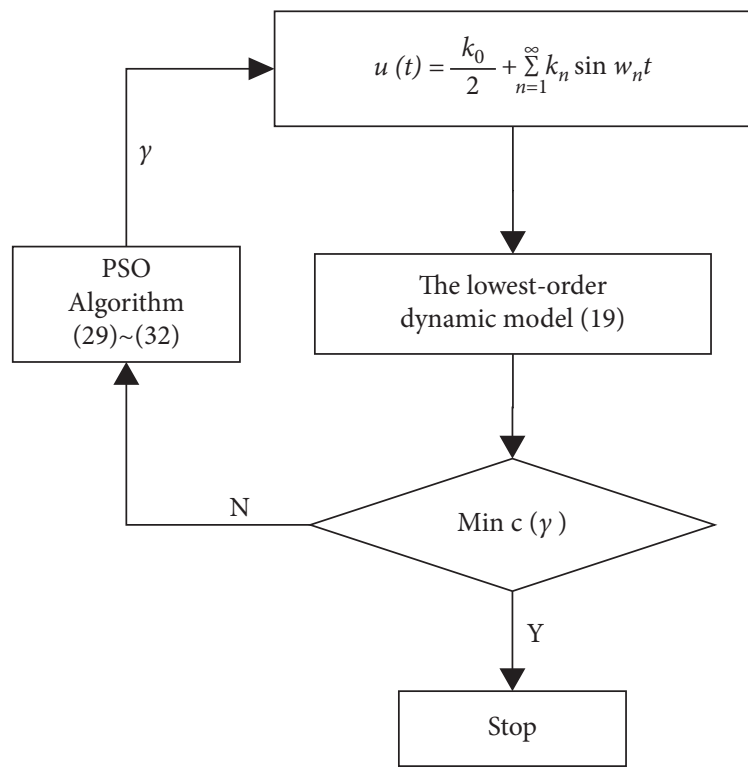

Figure 10: Optimization process diagram.

$$
\gamma=\left[k_{10}, k_{11}, \ldots \ldots, k_{19}, k_{20}, k_{21}, \ldots \ldots, k_{29}\right] \in R^{20} .
$$

When $\gamma$ is determined, the input signals applied to Joint 1 and Joint 2 can also be determined. Using equations (21)-(24), the relationship between the end vibration of the rigid-flexible mechanical arms and the applied torque of each joint is established. Therefore, the displacement of the end vibration of the flexible mechanical arm can be shown by the undetermined parameter $\gamma$ as follows:

$$
w(t, \gamma)=\sum_{i=1}^{N} \Gamma_{i}(x) q_{i}(t, \gamma)
$$

Moreover, the final target position of the mechanical arms after applying the input signal (25) is supposed to be $\left(\sigma_{t f}, \delta_{t f}\right)$, and the end position error function of the flexible mechanical arm in the movement is built as follows:

$$
e_{p}(t, \gamma)=\sqrt{\left[\sigma_{t f}-\sigma(t, \gamma)\right]^{2}+\left[\delta_{t f}-\delta(t, \gamma)\right]^{2}} .
$$

The objective of this study is to minimize the error of the mechanical arms reaching the target position and the residual vibration displacement at the end of the mechanical arms. The objective optimization function is established as follows:

$$
c(\gamma)=\xi_{1}|w(t, \gamma)|+\xi_{2} e_{p}(t, \gamma),
$$

where $\xi_{1}$ and $\xi_{2}$ are two weighted coefficients, and $\xi_{1}+\xi_{2}=1$. The objective of the optimization is to minimize the objective function (30) by adjusting these parameters to be optimized. In this way, the precise positioning of the end position of the mechanical arms and the suppression of residual vibration can be transformed into solving the optimal value of each parameter in equation (27).

The PSO algorithm [46] is a study based on predation behavior of birds proposed by Kennedy et al. [46], because the algorithm is simple in structure and easy to implement. It is extensively applied since it can remember the current individual optimal and global optimal and requires only a few adjustment parameters [53]. It is a global optimization algorithm of probability type. The advantage of the nondeterministic algorithm is that the algorithm has more chances to solve the global optimal solution. It does not depend on the strict mathematical properties of the optimization problem itself. The PSO algorithm is applied to optimize the parameters in equation (27). In a searching space with $N$-dimension, let the quantity of particles be $m$, where the position of the $i$ th particle is $x_{i} \in R^{n}$, and its velocity is $v_{i} \in R^{n}$.pbest ${ }_{i} \in R^{n}$ represents the best position of the $i$ th particle.gbest ${ }_{i} \in R^{n}$ represents the best position of all particles. The velocity and position of the particles are updated as follows [46]:

$$
\left\{\begin{array}{l}
\left.v_{i}^{n+1}=v_{i}^{n}+c_{1} r_{1}\left(\text { pest }_{i}^{n}-x_{i}^{n}\right)\right)+c_{2} r_{2}\left(\text { g best }_{i}^{n}-x_{i}^{n}\right), \\
x_{i}^{n+1}=x_{i}^{n}+v_{i}^{n+1}, \quad(n=1,2, \ldots, N, i=1,2, \ldots, m),
\end{array}\right.
$$

where $c_{1}$ and $c_{2}$ are the contraction factors and $r_{1}$ and $r_{2}$ are the arbitrary values between $[-1,1]$. The detailed steps of parameter optimization based on particle swarm optimization for the rigid-flexible mechanical arms are as follows.

Step 1. In these solutions space, 20-dimensional particles are initialized as $\left\{x_{1}^{l}, x_{2}^{l}, \ldots, x_{N}^{l}\right\}$, and their corresponding flight velocities are recorded as $\left\{v_{1}^{l}, v_{2}^{l}, \ldots, v_{N}^{l}\right\}$.

Step 2. Each particle is substituted into equation (26), and the input signals of Joint 1 and Joint 2 are calculated. Then, the modal coordinates of the flexible mechanical arm are calculated according to equation (6). Subsequently, the displacement of end vibration is calculated according to equations (21)-(23) and (28). Finally, the fitness value of 


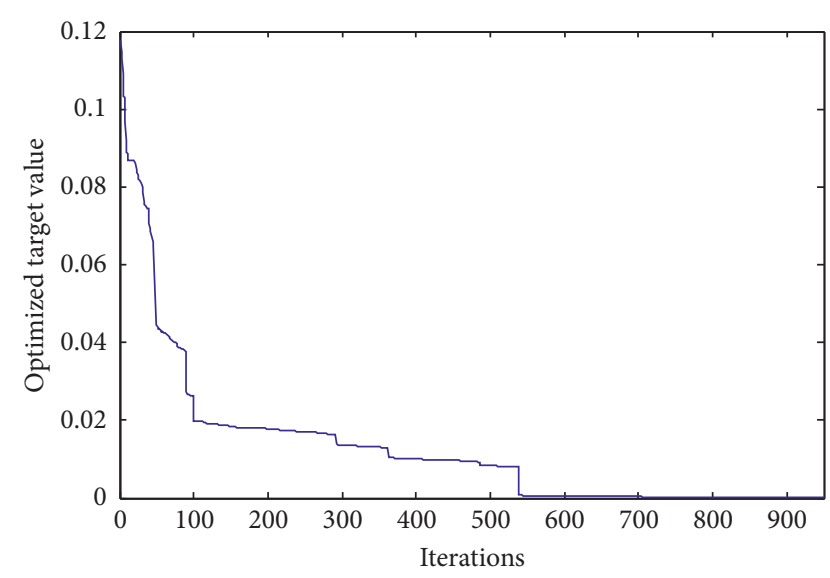

FIGURE 11: Optimization curve of the optimal objective function.

each particle is calculated according to the following equation:

$$
f_{i}(l)=c\left(x_{i}^{l}\right), \quad i=1,2, \ldots, N
$$

Step 3. The local optimal values and the globally optimal values are initialized, and the local optimal and the global optimal positions are determined at the same time. If $l=1$, then

$$
\left\{\begin{array}{l}
f_{p-\text { best }}=f_{g-\text { best }} \\
P_{p}=P_{g}
\end{array}\right.
$$

Step 4. The local optimal and the global optimal positions are recorded. The position and flight speed of particles are updated in accordance with the following formula:

$$
\left\{\begin{array}{l}
x_{i N}^{l+1}=x_{i N}^{l}+v_{i N}^{l+1}, \\
v_{i N}^{l+1}=v_{i N}^{l}+c_{1} r_{1}\left(p_{p N}^{l}-x_{i N}^{l}\right)+c_{2} r_{2}\left(p_{g N}^{l}-x_{i N}^{l}\right) .
\end{array}\right.
$$

Step 5. Continue to execute the program until the minimum value set by the optimization goal or the maximum number of iterations is met.

\subsection{Parameter Optimization Based on the PSO Algorithm.} Table 1 depicts the parameters of the rigid-flexible mechanical arms. The time of the input signal acting on Joint 1 and Joint 2 is $4 \mathrm{~s}$. The parameter setting of the PSO algorithm is as follows: $c_{1}$ and $c_{2}$ are constant values, which are set by 2.25; $r_{1}$ and $r_{2} \in[-1,1]$, where $r_{1}$ is -0.3 and $r_{2}$ is 0.5 . The particle quantity is 60 , the maximum iterations are 950 , and the minimum value of the optimization function is set by $10^{-5}$. The weight of the optimization function is set by $\xi_{1}=0.43$ and $\xi_{2}=0.57$ to ensure that the mechanical arm can reach the target position.

The curve of the optimization process based on the above algorithm is shown in Figure 11. It decreases monotonically until meeting the conditions when the algorithm stops. In this study, the objective function value is already less than the minimum setting value when the number of iterations reaches 540 generations. The final parameters optimized by the PSO algorithm are depicted in Table 2. The complete input signal is depicted in Figures 12(a) and 12(b). The optimized input signal is similar to the original input signal, but the transition of the optimized input signal is smoother.

The original input signal and the optimized input signal are applied to the rigid-flexible mechanical arms to compare the end vibration of the flexible mechanical arm. The movement of the end of the mechanical arms is depicted in Figures 13(a) and 13(b). After applying the original input signal for 4 seconds, the maximum amplitude of the residual vibration is $16.12 \mathrm{~mm}$. After applying the optimized input signal for 4 seconds, the end position error is $0.56 \mathrm{~mm}$, the maximum amplitude of the terminal residual vibration is $4.76 \mathrm{~mm}$, and the end residual vibration decreased by $70.22 \%$. This process not only realizes to suppress the residual vibration of the end of the mechanical arms obviously but also realizes to control the end position of the mechanical arms accurately and the improvement of the positioning accuracy of the mechanical arms.

\section{Experimental Verification}

A hardware experimental device of rigid-flexible mechanical arms is developed so as to verify the effectiveness of the method of dimension reduction. The sensors consist of three resistance strain gauges, which can measure the deformation of different parts accurately. For the accurate measurement of the strain force, the connection mode of the half bridge power supply circuit of the mechanical arms is selected. The strain force amplifier is used to accurately measure the voltage signal and amplify the measured voltage signal. Then, the fast data acquisition instrument is used for data acquisition and $\mathrm{A} / \mathrm{D}$ conversion for the collected data. Finally, the terminal deformation of the mechanical arms is calculated by computer, as shown in Figure 14.

According to the software of the control card, a pulse signal with a width of 5 seconds is given to the servo motor. According to the motor encoder, the angular velocity of the motor is obtained, and observing the voltage value of the resistance strain gauge, then can obtain the deformation of the end of the flexible arm through transformation. The simulation results of the reduced dimension model are compared with the experimental results, as depicted in Figures 15 and 16.

(1) As depicted in Figure 15, the simulation conclusions of the angular velocity of the flexible mechanical arm are mainly accordant with the experimental conclusions, which demonstrate that the selection of the spectral method and the dimension reduction method of Galerkin truncation theory can accurately reflect the characteristics of the dynamic model of the flexible mechanical arm.

(2) As depicted in Figure 16, in the first 5 seconds, the simulation conclusions of the first-order model by AIM are basically consistent with those of the 
TABLE 2: Coefficients optimized by the PSO algorithm.

\begin{tabular}{|c|c|c|c|}
\hline \multicolumn{2}{|c|}{ Joint 1 signal } & \multicolumn{2}{|c|}{ Joint 2 signal } \\
\hline$k_{10}=0.4135$ & $k_{15}=0.0540$ & $K_{20}=0.4152$ & $k_{25}=0.0613$ \\
\hline$k_{11}=0.2684$ & $k_{16}=0.0006$ & $k_{21}=0.2757$ & $k_{26}=0.0034$ \\
\hline$k_{12}=0.0003$ & $k_{17}=0.0375$ & $k_{22}=0.0006$ & $k_{27}=0.0406$ \\
\hline$k_{13}=0.0886$ & $k_{18}=0.0019$ & $k_{23}=0.0950$ & $k_{28}=0.0002$ \\
\hline$k_{14}=0.0004$ & $k_{19}=0.0301$ & $k_{24}=0.0004$ & $k_{29}=0.0308$ \\
\hline
\end{tabular}

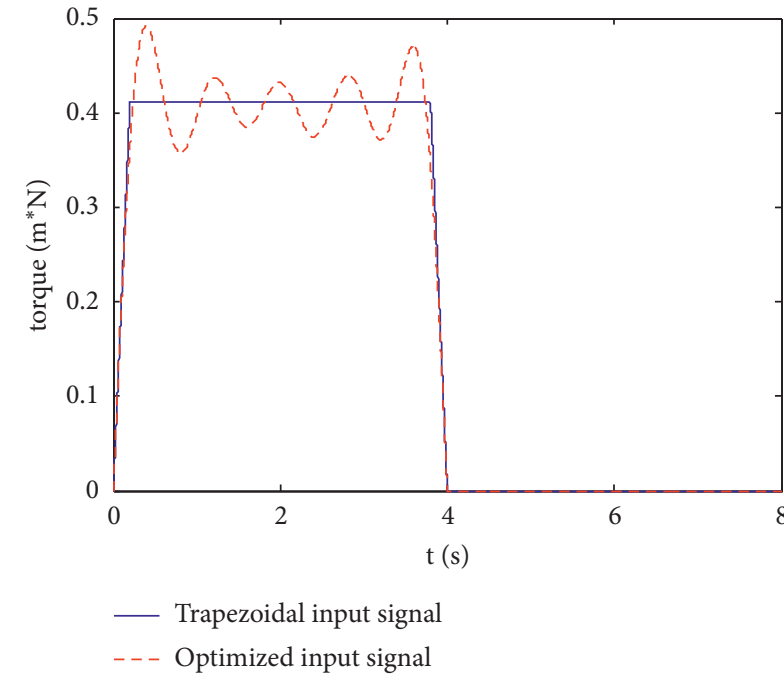

(a)

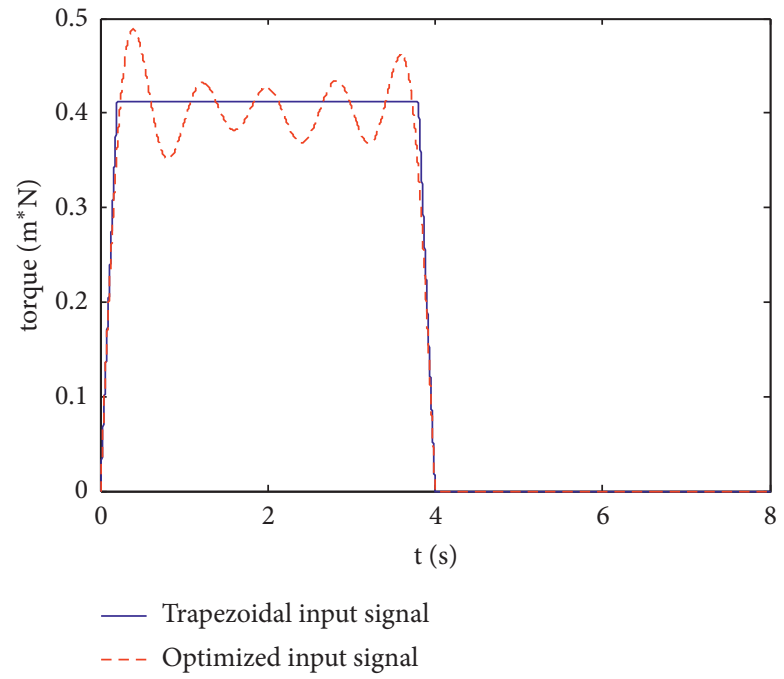

(b)

FIgURE 12: Comparison of the input signal: (a) Joint 1 input signal; (b) Joint 2 input signal.

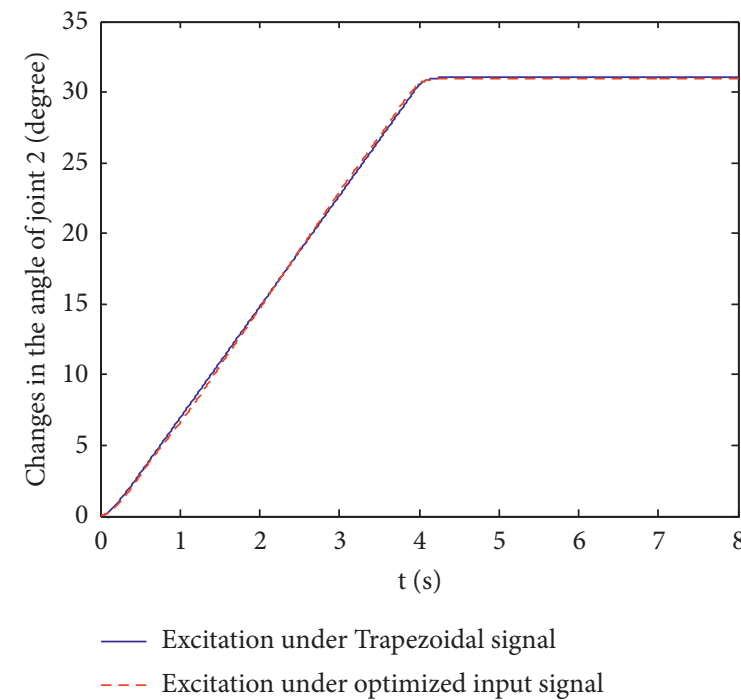

(a)

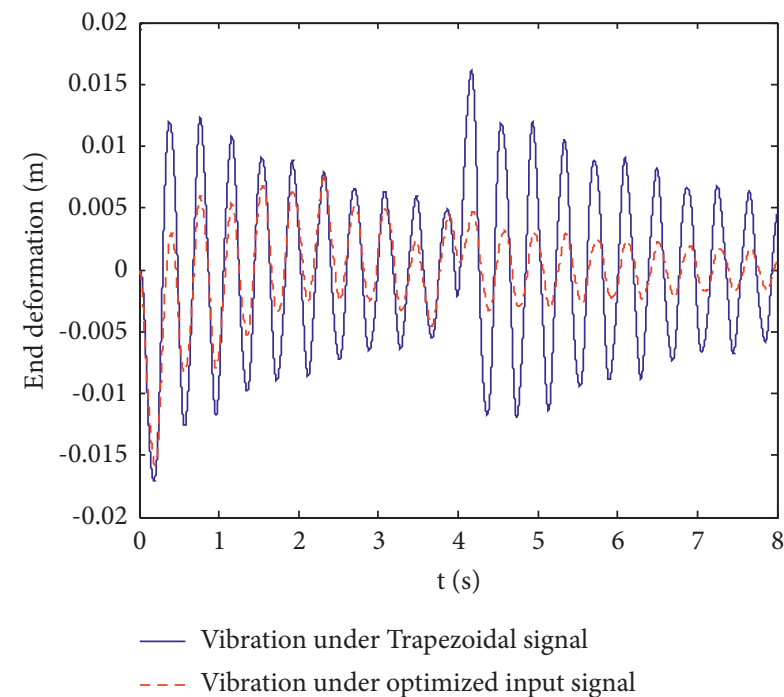

(b)

Figure 13: Comparison of end angle and end vibration: (a) end angle; (b) end vibration.

directly truncated third-order modal model. When the voltage signal is removed after the fifth second, the vibration mutation in the terminal deformation is removed slowly under the effect of structural damping, which confirms that the overall goal can further simplify the third-order system and lays a foundation for the next research on the control of rigid-flexible mechanical arms. 


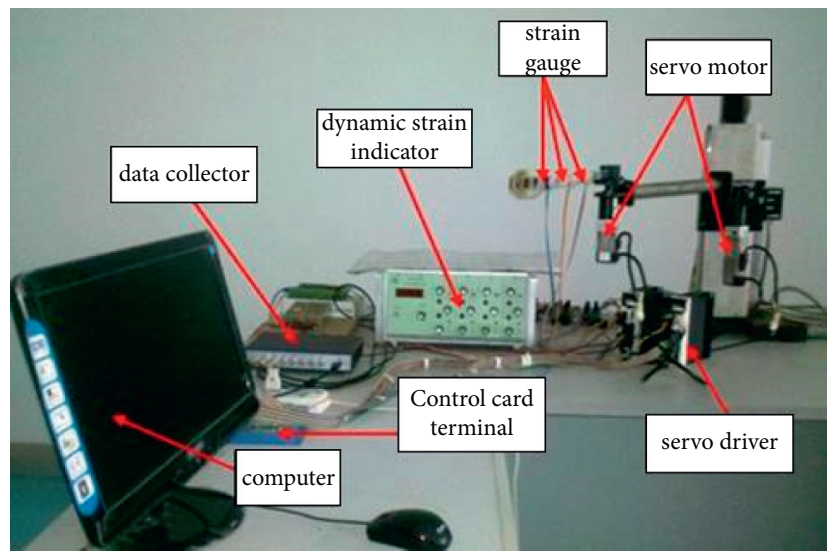

FIgURE 14: Experiment platform.

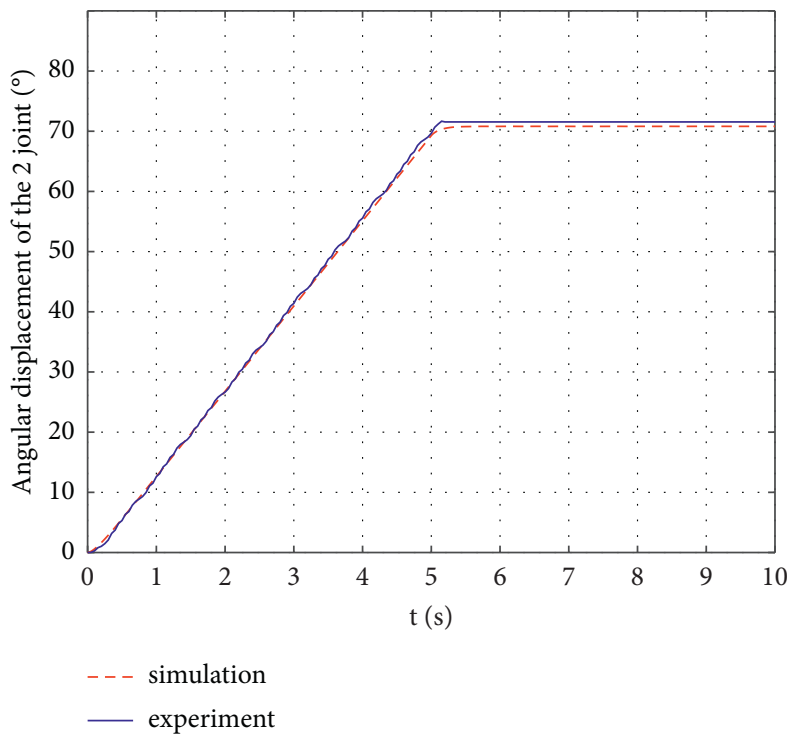

FIGURE 15: Comparison of flexible joint angular displacement.

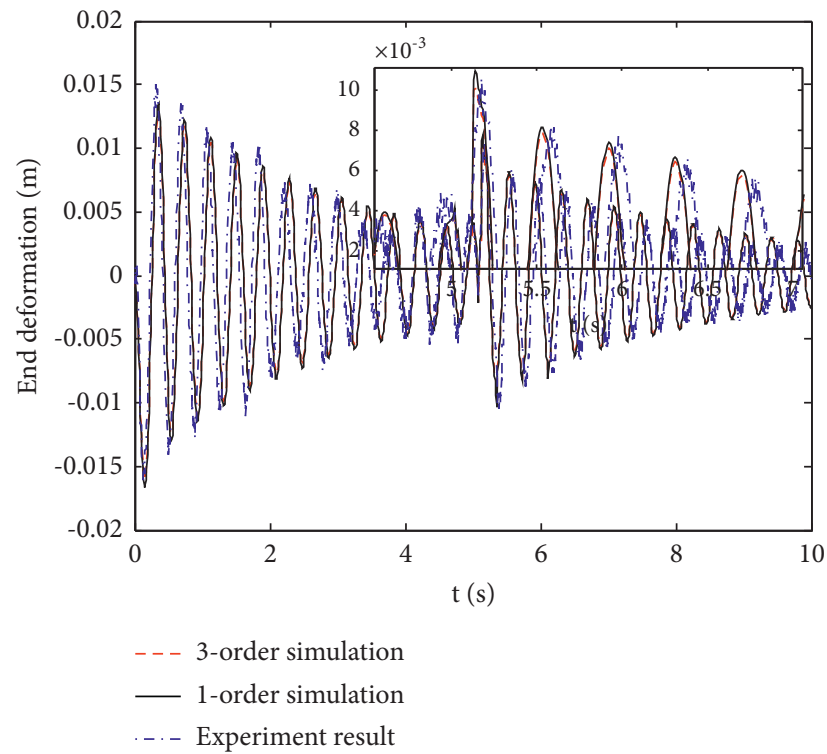

Figure 16: Comparison of deformation of flexible joint end. 
(3) The experimental verification of vibration suppression is underway.

\section{Conclusion}

A dynamic model reduction method of rigid-flexible mechanical arms based on the approximate inertial manifold and a new method of terminal residual vibration suppression based on particle swarm optimization feedforward control are established. Firstly, the approximate inertial manifold theory is used to approximate the model of the rigid-flexible mechanical arms whose high-order mode of TGM is truncated and acts on the low-order mode, and its low-order system dynamics model is obtained. Then, the feedforward control method based on particle swarm optimization is applied to the dynamic model of the lowest-order rigid-flexible mechanical arm. The particle swarm optimization algorithm is used to modify the amplitude and frequency of the input signal composed of a limited number of sinusoidal signals with uncertain parameters so as to reduce the target position error and end residual vibration. The simulation results show that compared with the original input signal before optimization, the maximum amplitude of end residual vibration of rigid-flexible mechanical arms is reduced by $70 \%$. Under the action of optimizing the input signal, the target position error is relatively small in the specified operation cycle. Simulation results show that this method can achieve accurate positioning and improve the suppression effect of residual vibration. The simulation results of angular displacement and end vibration of the simplified model are basically consistent with the experimental results of the hardware platform. The future research work is to verify the feedforward control method based on particle swarm optimization on the experimental platform and compare it with the simulation results to verify the effectiveness of the method [54].

\section{Appendix}

\section{$\mathbf{A}$}

$$
\begin{aligned}
& A_{11}=J_{1}+J_{t}+M_{t} l_{1}^{2}+\ell_{2} l_{1}^{2} l_{2}+\frac{1}{3} l_{2} l_{2}^{3}+M_{p}\left(l_{1}^{2}+l_{2}^{2}+2 l_{1} l_{2} \cos \theta_{2}+w^{2}-2 l_{1} w \sin \theta_{2}\right) \\
& +\ell_{2} l_{1}^{2} l_{2} \cos \theta_{2}+\ell_{2} \int_{0}^{l_{2}} w^{2} \mathrm{~d} x-2 \ell_{2} l_{1} \sin \theta_{2} \int_{0}^{l_{2}} w \mathrm{~d} x \\
& A_{12}=A_{21}=J_{t}+\frac{1}{3} \ell_{2} l_{2}^{3}+M_{p}\left(l_{2}^{2}+l_{1} l_{2} \cos \theta_{2}+w^{2}-l_{1} w \sin \theta_{2}\right)+\frac{1}{2} \ell_{2} l_{1} l_{1}^{2} \cos \theta_{2}+\ell_{2} \int_{0}^{l_{2}} w^{2} \mathrm{~d} x-\ell_{2} l_{1} \sin \theta_{2} \int_{0}^{l_{2}} w \mathrm{~d} x, \\
& A_{22}=J_{t}+\frac{1}{3} \ell_{2} l_{2}^{3}+M_{p}\left(l_{2}^{2}+w^{2}\right)+\ell_{2} \int_{0}^{l_{2}} w^{2} \mathrm{~d} x \\
& G_{1}\left(\dot{\theta}_{1}, \dot{\theta}_{2}, \theta_{2}\right)=-\left(\frac{1}{2} \ell_{2} l_{1} l_{2}^{2} \sin \theta_{2}+M_{p}\left(l_{1} l_{2} \sin \theta_{2}+l_{1} w \cos \theta_{2}\right)+\ell_{2} l_{1} \cos \theta_{2} \int_{0}^{l_{2}} w \mathrm{~d} x\right) \dot{\theta}_{2}^{2} \\
& +\left(-\left[\ell_{2} l_{1} l_{2}^{2} \sin \theta_{2}+2 \ell_{2} l_{1} \cos \theta_{2}+M_{p}\left(2 l_{1} l_{2} \sin \theta_{2}+2 l_{1} w \cos \theta_{2}\right)+\ell_{2} l_{1} \cos \theta_{2} \int_{0}^{l_{2}} w \mathrm{~d} x\right]\right) \dot{\theta}_{1} \dot{\theta}_{2} \\
& +M_{p}\left(l_{2}^{2}+2 w \cdot \dot{w}-2 l_{1} \dot{w} \sin \theta_{2}\right)+2 \ell_{2} \int_{0}^{l_{2}} w \cdot \dot{w} d x-2 \ell_{2} l_{1} \sin \theta_{2} \int_{0}^{l_{2}} \dot{w} d x\left(\dot{\theta}_{1}+\dot{\theta}_{2}\right)+M_{p} w \cdot\left(l_{1} \cos \theta_{2}+l_{2}\right) \\
& +\ell_{2} l_{1} \cos \theta_{2} \int_{0}^{l_{2}} w \mathrm{~d} x+\ell_{2} \int_{0}^{l_{2}} x \cdot w \mathrm{~d} x
\end{aligned}
$$




$$
\begin{aligned}
G_{2}\left(\dot{\theta}_{1}, \dot{\theta}_{2}, \theta_{2}\right)= & -\left(\frac{1}{2} \ell_{2} l_{1} l_{2}^{2} \sin \theta_{2}+M_{p}\left(l_{1} l_{2} \sin \theta_{2}+l_{1} w \cos \theta_{2}\right)+\ell_{2} l_{1} \cos \theta_{2} \int_{0}^{l_{2}} w \mathrm{~d} x\right) \dot{\theta}_{2}^{2} \\
& +M_{p}\left(l_{2}^{2}+w \cdot \dot{w}\right)+2 \ell_{2} \int_{0}^{l_{2}} w \cdot \dot{w} \mathrm{~d} x\left(\dot{\theta}_{1}+\dot{\theta}_{2}\right)+M_{p} w \cdot\left(l_{1} \cos \theta_{2}+l_{2}\right),
\end{aligned}
$$

where $w$ replaces $w(x, t)$.

B

$$
\begin{aligned}
& m_{11} \quad m_{12} \quad m_{13} \quad m_{14} \quad m_{15} \\
& m_{12} \quad m_{22} \quad m_{23} \quad m_{24} \quad m_{25} \\
& M=m_{13} \quad m_{23} \quad m_{33} \quad 0 \quad 0 \quad \text {, } \\
& m_{14} \quad m_{24} \quad 0 \quad m_{44} \quad 0 \\
& m_{15} m_{25} \quad 0 \quad 0 \quad m_{55} \\
& m_{11}=J_{1}+J_{t}+M_{t} l_{1}^{2}+\ell_{2} l_{1}^{2} l_{2}+\frac{1}{3} \ell_{2} l_{2}^{3}+\ell_{2} l_{1}^{2} l_{2} \cos \theta_{2}+\ell_{2} l_{2} \sum_{i=1}^{3} q_{i}^{2}(t)-2 \ell_{2} l_{1} \sin \theta_{2} \sum_{i=1}^{3} \int_{0}^{l_{2}} \Gamma_{i}(x) q_{i}(t) \mathrm{d} x \\
& +M_{p} l_{1}^{2}+l_{2}^{2}+2 l_{1} l_{2} \cos \theta_{2}-2 \ell_{2} l_{1} \sin \theta_{2} \sum_{i=1}^{3} \int_{0}^{l_{2}} \Gamma_{i}(x) q_{i}(t) \mathrm{d} x+\left(\sum_{i=1}^{3} \int_{0}^{l_{2}} \Gamma_{i}(x) q_{i}(t) \mathrm{d} x^{2}\right) \\
& m_{12}=J_{t}+\frac{1}{3} \ell_{2} l_{2}^{3}+\frac{1}{2} \ell_{2} l_{1} l_{2}^{2} \cos \theta_{2}+\ell_{2} \sum_{i=1}^{3} q_{i}^{2}(t)-\ell_{2} l_{1} \sin \theta_{2} \sum_{i=1}^{3} \int_{0}^{l_{2}} \Gamma_{i}(x) q_{i}(t) \mathrm{d} x+M_{p} l_{2}^{2} \\
& +\left(l_{1} l_{2} \cos \theta_{2}+\left(\sum_{i=1}^{3} \int_{0}^{l_{2}} \Gamma_{i}(x) q_{i}(t) \mathrm{d} x\right)^{2}-l_{1} \sin \theta_{2} \sum_{i=1}^{3} \int_{0}^{l_{2}} \Gamma_{i}(x) q_{i}(t) \mathrm{d} x\right) \\
& m_{22}=J_{t}+\frac{1}{3} \rho_{2} l_{2}^{3}+\rho_{2} l_{2} \sum_{i=1}^{3} q_{i}^{2}(t)+M_{p}\left(l_{2}^{2}+\left(\sum_{i=1}^{3} \int_{0}^{l_{2}} \Phi_{i}(x) q_{i}^{2}(t) \mathrm{d} x\right)^{2}\right) m_{1(i+2)} \\
& =\rho_{2} \int_{0}^{l_{2}} x \cdot \Phi_{i}(x) \mathrm{d} x+\rho_{2} l_{1} \cos \theta_{2} \int_{0}^{l_{2}} \Phi_{i}(x) \mathrm{d} x+M_{p} l_{1} \cos \theta_{2} \int_{0}^{l_{2}} \Phi_{i}(x) \mathrm{d} x, \quad i=1,2,3, \\
& m_{2(i+2)}=\rho_{2} \int_{0}^{l_{2}} x \cdot \Phi_{i}(x) \mathrm{d} x+M_{p} l_{2} \int_{0}^{l_{2}} \Phi_{i}(x) \mathrm{d} x, \quad i=1,2,3, \\
& m_{(i+2)(i+2)}=\rho_{2} \int_{0}^{l_{2}} \Phi_{i}(x) \mathrm{d} x, \quad i=1,2,3,
\end{aligned}
$$




$$
\begin{aligned}
& K=\left[\begin{array}{ccccc}
0 & 0 & 0 & 0 & 0 \\
0 & 0 & 0 & 0 & 0 \\
0 & 0 & k_{3} & 0 & 0 \\
0 & 0 & 0 & k_{4} & 0 \\
0 & 0 & 0 & 0 & k_{5}
\end{array}\right], \\
& k_{i+2}=E I_{2}\left(H_{i}^{0}\right)^{4}, \quad i=1,2,3 \\
& h_{i}(1)=\int_{0}^{l_{2}} \Gamma_{i}(x) \mathrm{d} x \text {, } \\
& h_{i}(x)=\int_{0}^{l_{2}} x \cdot \Gamma_{i}(x) \mathrm{d} x, \quad i=1,2,3, \\
& F=\left[\begin{array}{lllll}
f_{1} & f_{2} & f_{3} & f_{4} & f_{5}
\end{array}\right]^{T} \\
& F_{1}(\theta, q, \dot{\theta}, \dot{q})=\left[\begin{array}{ll}
f_{1} & f_{2}
\end{array}\right]^{T}, \\
& F_{2}(\theta, q, \dot{\theta}, \dot{q})=\left[\begin{array}{lll}
f_{3} & f_{4} & f_{5}
\end{array}\right]^{T} \text {. }
\end{aligned}
$$

\section{Data Availability}

The simulation and experimental data used to support the findings of this study are available from the corresponding author upon request.

\section{Conflicts of Interest}

The authors declare that there are no conflicts of interest regarding the publication of this paper.

\section{Acknowledgments}

This work was supported in part by the National Key Research and Development Project under Grant 2018YFB1307203 and the Project of State Key Laboratory of High Performance Complex Manufacturing, Central South University, under Grant ZZYJKT2021-17.

\section{References}

[1] Z. Zhao, X. He, and C. K. Ahn, "Boundary disturbance observer-based control of a vibrating single-link flexible mechanical arm," IEEE Transactions on Systems, Man, and Cybernetics, vol. 49, no. 5, pp. 1-9, 2019.

[2] H. Karagülle, L. Malgaca, M. Dirilmiş, M. Akdağ, and Ş. Yavuz, "Vibration control of a two-link flexible manipulator," Journal of Vibration and Control, vol. 23, no. 12, pp. 2023-2034, 2015.

[3] D. Liang, Y. Song, T. Sun, and X. Jin, "Rigid-flexible coupling dynamic modeling and investigation of a redundantly actuated parallel manipulator with multiple actuation modes," Journal of Sound and Vibration, vol. 403, no. 9, pp. 129-151, 2017.

[4] W. Congqing, W. Pengfei, and Z. Xin, "Composite sliding mode control for a free-floating space rigid-flexible coupling mechanical arm system," International Journal of Advanced Robotic Systems, vol. 124, no. 10, pp. 1-10, 2013.

[5] S. Gharooni, B. Heller, and M. O. Tokhi, "A new hybrid spring brake orthosis for controlling hip and knee flexion in the swing phase," IEEE Transactions on Neural Systems and Rehabilitation Engineering, vol. 9, no. 1, pp. 106-107, 2001.

[6] R. Kumar, P. Berkelman, and P. Gupta, "Preliminary experiments in cooperative human/robot force control for robot assisted microsurgical manipulation," in Proceedings of the IEEE International Conference on Robotics and Automation (ICRA), pp. 610-617, Montreal, Canada, January 2000.

[7] Z. Ni, J. Liu, Z. Wu, and X. Shen, "Identification of the statespace model and payload mass parameter of a flexible space manipulator using a recursive subspace tracking method," Chinese Journal of Aeronautics, vol. 32, no. 2, pp. 513-530, 2019.

[8] D. Meng, X. Wang, W. Xu, and B. Liang, "Space robots with flexible appendages: dynamic modeling, coupling measurement, and vibration suppression," Journal of Sound and Vibration, vol. 396, no. 5, pp. 30-50, 2017.

[9] H. N. Rahimi and M. Nazemizadeh, "Dynamic analysis and intelligent control techniques for flexible manipulators: a review," Advanced Robotics, vol. 28, no. 2, pp. 63-76, 2014.

[10] X. Zhou, H. Wang, Y. Tian, and G. Zheng, "Disturbance observer-based adaptive boundary iterative learning control for a rigid-flexible manipulator with input backlash and endpoint constraint," International Journal of Adaptive Control and Signal Processing, vol. 34, no. 9, pp. 1220-1241, 2020.

[11] Y. Zhao, C. M. Ruan, and S. .W. Wang, "Accurate modeling and experimental research on the second-order theory of rigid-flexible flexible mechanical arms," Chinese Journal of Mechanical Engineering, vol. 29, no. 2, pp. 205-210, 2018.

[12] D. Liang, Y. Song, T. Sun, and X. Jin, "Dynamic modeling and hierarchical compound control of a novel 2-DOF flexible parallel manipulator with multiple actuation modes," $\mathrm{Me}$ chanical Systems and Signal Processing, vol. 103, no. 3, pp. 413-439, 2018.

[13] F. Cao and J. Liu, "Three-dimensional modeling and input saturation control for a two-link flexible manipulator based on infinite dimensional model," Journal of the Franklin Institute, vol. 357, no. 2, pp. 1026-1042, 2020.

[14] G. Piras, W. L. Cleghorn, and J. K. Mills, "Dynamic finiteelement analysis of a planar high-speed, high-precision 
parallel manipulator with flexible links," Mechanism and Machine Theory, vol. 40, no. 7, pp. 849-862, 2005.

[15] C. Sun, W. He, and J. Hong, "Neural network control of a flexible robotic mechanical arm using the lumped springmass model," IEEE Transactions on Systems, Man, and Cybernetics, vol. 47, no. 8, pp. 1863-1874, 2016.

[16] M. Vakil, R. Fotouhi, and P. N. Nikiforuk, "Trajectory tracking for the end-effector of a class of flexible link manipulators," Journal of Vibration and Control, vol. 17, no. 1, pp. 55-68, 2011.

[17] X. Xiaoping Zhang, W. Wenwei Xu, and V. Chellaboina, "PDE modeling and control of a flexible two-link manipulator," IEEE Transactions on Control Systems Technology, vol. 13, no. 2, pp. 301-312, 2005.

[18] G. Natasha, S. Suharjito, and V. Noviantri, "Saint-venant model analysis of trapezoidal open channel water flow using finite difference method," Procedia Computer Science, vol. 157, no. 11, pp. 6-15, 2019.

[19] M. Jiang and H. Deng, "Optimal combination of spatial basis functions for the model reduction of nonlinear distributed parameter systems," Communications in Nonlinear Science and Numerical Simulation, vol. 17, no. 12, pp. 5240-5248, 2012.

[20] L. Chen and H. Deng, "Model reduction of rigid-flexible manipulators with experimental validation," Advanced $M a-$ terials Research, vol. 655-657, no. 1, pp. 1101-1107, 2013.

[21] Y. C. Zhou, Z. Lu, H. Hu, and Y. S. Hu, "Surrogate modeling of high-dimensional problems via data-driven polynomial chaos expansions and sparse partial least square," Computer Methods in Applied Mechanics and Engineering, vol. 364, no. 1, pp. 1-24, 2020.

[22] J.-Z. Zhang, Y. Liu, and P.-H. Feng, "Approximate inertial manifolds of Burgers equation approached by nonlinear Galerkin's procedure and its application," Communications in Nonlinear Science and Numerical Simulation, vol. 16, no. 12, pp. 4666-4670, 2011.

[23] K. Ito and K. Kunisch, "Reduced-order optimal control based on approximate inertial manifolds for nonlinear dynamical systems," SIAM Journal on Numerical Analysis, vol. 46, no. 6, pp. 2867-2891, 2008.

[24] S. Hidetsugu, "Chaotic dynamics of an unstable Burgers equation,” Physica D, vol. 129, no. 2, pp. 57-67, 1999.

[25] B. Mario, S. Viriato, and C. Francisco, "Dynamics and synchronization of numerical solutions of the Burgers equation," Computational and Applied Mathematics, vol. 231, no. 2, pp. 793-806, 2009.

[26] A. Abe, "Trajectory planning for residual vibration suppression of a two-link rigid-flexible manipulator considering large deformation," Mechanism and Machine Theory, vol. 44, no. 9, pp. 1627-1639, 2009.

[27] C. T. Kiang, A. Spowage, and C. K. Yoong, "Review of control and sensor system of flexible manipulator," Journal of Intelligent and Robotic Systems, vol. 77, no. 1, pp. 187-213, 2015.

[28] J. S. Lane and S. L. Dickerson, "Contribution of passive damping to the control of flexible mechanical arm," in Proceedings of the IEEE International Conference on Robotics and Automation (ICRA), pp. 175-180, Montreal, Canada, January 1984.

[29] Z.-c. Qiu, C. Li, and X.-m. Zhang, "Experimental study on active vibration control for a kind of two-link flexible manipulator," Mechanical Systems and Signal Processing, vol. 118, no. 7, pp. 623-644, 2019.

[30] E. Lu, W. Li, X. Yang, Y. Wang, and Y. Liu, "Optimal placement and active vibration control for piezoelectric smart flexible manipulators using modal $\mathrm{H} 2$ norm," Journal of Intelligent Material Systems and Structures, vol. 29, no. 11, pp. 2333-2343, 2018.

[31] S. Zhang, Y. Zhang, and X. Zhang, "Fuzzy PID control of a two-link flexible mechanical arm," J. Vibroeng.vol. 18, no. 1, pp. 250-256, 2016.

[32] H.-J. Yang and M. Tan, "Sliding mode control for flexible-link manipulators based on adaptive neural networks," International Journal of Automation and Computing, vol. 15, no. 2, pp. 239-248, 2018.

[33] A. Izadbakhsh, "Robust control design for rigid-link flexiblejoint electrically driven robot subjected to constraint: theory and experimental verification," Nonlinear Dynamics, vol. 85, no. 2, pp. 751-765, 2016.

[34] J. O. Pedro and T. Tshabalala, "Hybrid NNMPC/PID control of a two-link flexible mechanical arm with actuator dynamics," in Proceedings of the Asian Control Conference (ASCC), Jeju Island, Korea, May 2015.

[35] X. Xing and J. Liu, "PDE model-based state-feedback control of constrained moving vehicle-mounted flexible manipulator with prescribed performance," Journal of Sound and Vibration, vol. 441, no. 2, pp. 126-151, 2019.

[36] Z. Li, B. Huang, Z. Ye, M. Deng, and C. Yang, "Physical human-robot interaction of a robotic exoskeleton by admittance control," IEEE Transactions on Industrial Electronics, vol. 65, no. 12, pp. 9614-9624, 2018.

[37] Z. Li, J. Li, S. Zhao, Y. Yuan, Y. Kang, and C. L. P. Chen, "Adaptive neural control of a kinematically redundant exoskeleton robot using brain-machine interfaces," IEEE Transactions on Neural Networks and Learning Systems, vol. 30, no. 12, pp. 3558-3571, 2019.

[38] Y. L. Liu, W. Su, Z. Li et al., "Motor-imagery-based teleoperation of a dual-arm robot performing manipulation tasks," IEEE Transactions on Cognitive and Developmental Systems, vol. 11, no. 3, pp. 414-424, 2019.

[39] H. Su, Y. Hu, H. R. Karimi, A. Knoll, G. Ferrigno, and E. De Momi, "Improved recurrent neural network-based manipulator control with remote center of motion constraints: experimental results," Neural Networks, vol. 131, pp. 291-299, 2020.

[40] H. Su, W. Qi, Y. Hu, H. R. Karimi, G. Ferrigno, and E. De Momi, "An incremental learning framework for human-like redundancy optimization of anthropomorphic manipulators," IEEE Transactions on Industrial Informatics, vol. 9, p. 1, 2020.

[41] H. Su, W. Qi, C. Yang, J. Sandoval, G. Ferrigno, and E. D. Momi, "Deep neural network approach in robot tool dynamics identification for bilateral teleoperation," IEEE Robotics and Automation Letters, vol. 5, no. 2, pp. 2943-2949, 2020.

[42] X. Zhou, W. Qi, S. E. Ovur et al., "A novel muscle-computer interface for hand gesture recognition using depth vision," Journal of Ambient Intelligence and Humanized Computing, vol. 11, no. 11, pp. 5569-5580, 2020.

[43] X. Y. Zhou, J. He, Q. He, C. Ren, and M. He, "Motion kinematics analysis of a horse inspired terrain-adaptive unmanned vehicle with four hydraulic swing mechanical arms," IEEE Access, vol. 8, no. 2, pp. 194350-194362, 2020.

[44] H. Yavuz, S. Mistıkoğlu, and S. Kapucu, "Hybrid input shaping to suppress residual vibration of flexible systems," Journal of Vibration and Control, vol. 18, no. 1, pp. 132-140, 2012.

[45] K. Shin and M. J. Brennan, "Two simple methods to suppress the residual vibrations of a translating or rotating flexible 
cantilever beam," Journal of Sound and Vibration, vol. 312, no. 1, pp. 140-150, 2007.

[46] Y.-x. Zheng and Y. Liao, "Parameter identification of nonlinear dynamic systems using an improved particle swarm optimization,” Optik, vol. 127, no. 19, pp. 7865-7874, 2016.

[47] R. Mandel, A. Shooshtari, and M. Ohadi, "A “2.5-D” modeling approach for single-phase flow and heat transfer in manifold microchannels," International Journal of Heat and Mass Transfer, vol. 126, no. 1, pp. 317-330, 2018.

[48] M. Jolly, I. Kevrekidis, and E. Titi, "Approximate inertial manifolds for the Kuramoto-Sivashinsky equation: analysis and computations," Physica D: Nonlinear Phenomena, vol. 44, no. 2, pp. 38-60, 1990.

[49] X. Li and C. Sun, "Inertial manifolds for the 3D modifiedLeray- $\alpha$ model," Journal of Differential Equations, vol. 268, no. 4, pp. 1532-1569, 2020.

[50] Y. Pan and H. Deng, "Model reduction of a two-link rigidflexible manipulators based on spectral appximation method." in Proceedings of the International Conference on Electrical Engineering, Computing Science and Automatic Control (ICEEAC), pp. 26-28, Mexico City, Mexico, November 2010.

[51] S. Baglioni, F. Cianetti, C. Braccesi, and D. M. De Micheli, "Multibody modelling of N DOF robot arm assigned to milling manufacturing. Dynamic analysis and position errors evaluation," Journal of Mechanical Science and Technology, vol. 30, no. 1, pp. 405-420, 2016.

[52] X. chen and Z. C. Fang, "Feedback control and numerical simulation of flexible mechanical arm vibration," Journal of Vibration and Shock, vol. 25, no. 1, pp. 1-4, 2006.

[53] D. H. Kim, "A swarm system design based on a modified particle swarm algorithm for a self-organizing scheme," Advanced Robotics, vol. 20, no. 8, pp. 913-932, 2006. 